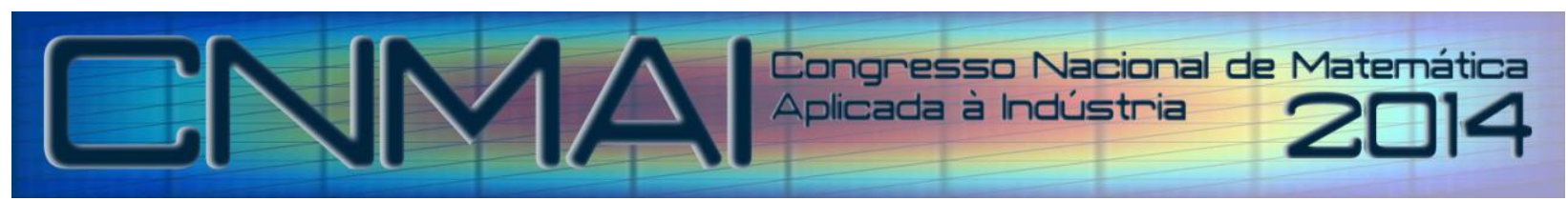

18 a 21 de novembro de 2014, Caldas Novas - Goiás

\title{
COMPARAÇÃO ENTRE MODELOS ESTATÍSTICOS APLICADOS À ANÁLISE DE VIBRAÇÕES EM TURBINAS EÓLICAS DE PEQUENO PORTE
}

\author{
José Rafael Bezerra Anastacio, jorafael_bezerra2@hotmail.com ${ }^{1}$ \\ Paulo Alexandre Costa Rocha, paulo.rocha@ufc.br ${ }^{1}$ \\ Francisco Marcilio Vieira Dantas, marciliodantas85@ gmail.com ${ }^{1}$ \\ Carla Freitas de Andrade, carla@ufc.br ${ }^{1}$ \\ Roberto de Araújo Bezerra, roberto.bezerra@gmail ${ }^{1}$ \\ ${ }^{1}$ Universidade Federal do Ceará - Centro de Tecnologia - Departamento de Engenharia Mecânica - Av. Humberto \\ Monte S/N, Campus do Pici, 63180-000, Fortaleza, Ceará, Brazil
}

\begin{abstract}
Resumo: Neste trabalho é feito um estudo comparativo entre métodos estatísticos aplicados à análise de vibrações em turbinas eólicas de pequeno porte. Foram abordadas duas metodologias: análise de vibrações no domínio do tempo e uma análise de flutuações. Para realizar a primeira, utilizou-se de ferramentas como média absoluta, média global, fator de crista, variância, assimetria, curtose e momento de sexta ordem. Já para a análise de flutuações, aplicou-se a detrended fluctuation analysis (DFA). Os sinais de vibração capturados foram obtidos através de testes em turbinas eólicas em escala reduzida que possuem rotores tripá, perfil NREL S809, de $40 \mathrm{~cm}$ de diâmetro, estes diferenciados entre si pela velocidade específica de projeto de suas pás ( $\lambda$ ). Os testes foram conduzidos em túnel de vento localizado no Laboratório de Aerodinâmica e Mecânica dos Fluidos (LAERO) da Universidade Federal do Ceará.
\end{abstract}

Palavras-chave: Turbina eólica, metódos estatisticos, análise de flutuações, controle de vibração.

\section{INTRODUÇÃ̃O}

No estudo de vibração em turbinas eólicas, fazem-se testes nos quais são capturados sinais de vibração que expressam valores de uma variável (deslocamento, velocidade ou aceleração). O processo observacional desta variável e sua consequente quantificação numérica gera uma sequência de dados distribuídos no tempo que é denominada série temporal. Há inúmeras ferramentas disponíveis para análise de séries temporais.

As primeiras ferramentas consideradas são aquelas usadas para uma análise de vibração no domínio do tempo: média absoluta, média global, fator de crista, variância, assimetria, curtose e momento de sexta ordem. Estas são ferramentas mais conhecidos e sua utilização na análise de vibração é mais difundida.

A análise no domínio do tempo, no entanto, não leva em consideração que o sinal capturado é uma série temporal que pode apresentar características complexas. Entre estas, podem-se citar correlação de longo alcance e a não estacionariedade. Uma opção para trabalhar com séries temporais que possuem as características acima citadas é utilizar ferramentas de análise de flutuações estatísticas como a detrended fluctuation analysis (DFA). O presente trabalho tem como objetivo compara o desempenho desta ferramenta em comparação à análise no domínio do tempo para análise de vibrações em turbinas eólicas.

\section{METODOLOGIA}

A seguir é apresentada a metodologia utilizada para desenvolvimento do presente trabalho de pesquisa. Basicamente, o projeto se divide em cinco etapas sucessivas: projeto e construção das pás do rotor, montagem do aparato experimental, ensaios de captura de sinais vibratórios e análise dos dados obtidos.

\subsection{Projeto das pás do rotor}

O perfil aerodinâmico utilizado nas pás foi o perfil NREL S809, este que é mostrado na Fig. 1. O perfil utilizado é projetado especialmente para turbinas de eixo horizontal e dispõe na literatura de uma ampla quantidade de dados experimentais para consulta. A disposição do rotor utilizado nos testes é do tipo de três pás, de raio (R) igual a $20 \mathrm{~cm}$. 


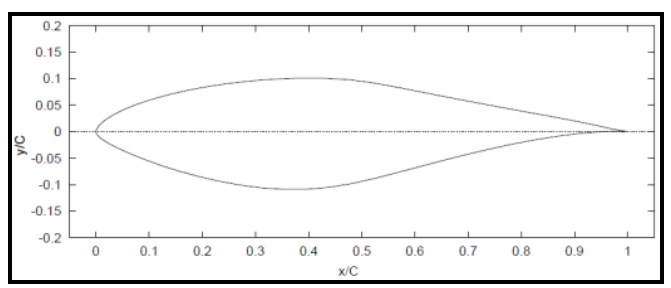

Figura 1. Perfil NREL S809. Fonte: Leishman (2006)

O projeto da pá de um rotor requer teoria aerodinâmica para sua concepção, já que os perfis aerodinâmicos são de geometria complexa. Cálculos são necessários para determinar parâmetros, como sua corda, (c), a espessura do perfil e distribuição de torção ( $\beta$ ) ao longo do comprimento da pá (Anastacio et al., 2013).

Para o projeto das pás será utilizada a teoria BEM (Blade Element Moment), pois esta teoria quando utilizada no projeto de pás resulta na geometria de máxima eficiência para um gerador eólico. Em suma, esta teoria é a junção de outras duas teorias existente: a teoria do momento unidimensional e a teoria do elemento de pá. Logo, para compreender toda a teoria BEM, faz-se necessário compreender alguns princípios das outras que a compõem.

Inicialmente o rotor eólico é modelado como um disco atuador que irá interferir no escoamento de ar com o objetivo de absorver parte da energia cinética do vento, convertendo-a em potência mecânica de eixo, que posteriormente será convertida em potência elétrica através de um gerador elétrico. A interferência é dividida em uma componente axial e uma tangencial, descritas pelos seus respectivos fatores: fator de interferência axial (a), que é a fração de velocidade axial que o fluido perde ao passar pela turbina, e o fator de interferência tangencial ( $a$ ') que é a fração da velocidade de rotação do rotor que é transferida para o fluido em forma de momento angular. (Rocha, et al. 2010).

Para cada posição radial, as componentes da velocidade $(W)$ podem ser determinadas como sendo uma resultante entre a velocidade do vento [U $(1-a)]$ e a velocidade de rotação do rotor $\left[\Omega r\left(1+a^{\prime}\right)\right]$ e dos fatores de interferência (Manwell et al., 2009). Um desenho esquemático do perfil aerodinâmico juntos com todas as componentes de velocidades é mostrado na Fig. 2.

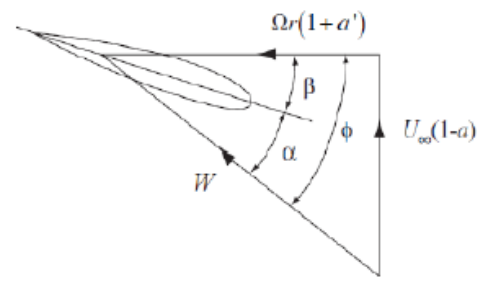

Figura 2. Ângulos no elemento de pá. Fonte: Burton (2001)

Assume-se que as forças nas pás de uma turbina eólica podem ser expressas como uma função dos coeficientes de sustentação e arrasto e sua resultante é a responsável pela variação da quantidade de movimento. A pá é dividida em $n$ seções de elementos e as seguintes premissas são aceitas (Manwell et al., 2009):

- Não há interação entre os elementos aerodinâmicos (portanto, sem fluxo radial);

- As forças sobre as pás são determinadas exclusivamente pelas características de sustentação e arrasto do perfil aerodinâmico da pá;

- Não há interferência tangencial, ou seja, o fator de indução tangencial ( $a^{\prime}$ ) é igual a zero;

- Não há força de arrasto, ou seja, o coeficiente de arrasto (Cd) é igual a 0 ;

- Não há perdas devido ao número finito de pás;

- O fator de indução axial, $a$, é igual a $1 / 3$.

O ângulo da velocidade relativa, $\phi$ é dado por (Burton et al., 2001):

$$
\tan \phi=\frac{1-\frac{1}{3}}{\lambda \mu\left(1+\frac{2}{9 \lambda^{2} \mu^{2}}\right)}
$$

O parâmetro $\lambda$ define a razão entre a velocidade da ponta da pá e a velocidade de corrente livre como mostrado na Eq. (2). Já a definição do parâmetro $\mu$ é a razão é mostrada na Eq. (3).

$$
\lambda=\frac{\Omega \mathrm{R}}{\mathrm{U}_{\infty}}
$$




$$
\mu=\frac{r}{R}
$$

onde $\Omega$ é definido como a velocidade angular da pá do rotor, $\mathrm{R}$ é o raio do rotor eólico e $\mathrm{U}_{\infty}$ é a velocidade de corrente livre no túnel de vento. A distribuição da corda, ao longo da pá, assumindo o método simplificado, é calculada pela seguinte expressão (Burton et al., 2001):

$$
c=\frac{2 \pi R \frac{8}{9}}{N \lambda C_{l} \sqrt{\left(1-\frac{1}{3}\right)^{2}+\lambda^{2} \mu^{2}\left[1+\frac{2}{9 \lambda^{2} \mu^{2}}\right]^{2}}}
$$

O ângulo de torção $\beta$ é obtido através da expressão (Burton et al., 2001):

$$
\beta=\phi-\alpha
$$

A geometria da pá é, então, completamente caracterizada tanto pela variação da corda (c) quanto pela variação do ângulo de torção $\beta$ ao longo da pá. Para que o projeto seja considerado um projeto ótimo, é necessário que a relação entre a força de sustentação (L) e a força de arrasto (D) seja máxima. Estas forças aerodinâmicas são representadas pelos parâmetros adimensionais $C_{L}$ e $C_{D}$, respectivamente.

A variação do ângulo de ataque $\alpha$ implica na mudança destes coeficientes, o que não é desejada. Como o ângulo $\phi$ varia ao longo da pá, varia-se o valor de $\beta$ de modo que ângulo de ataque $\alpha$ permaneça constante. Motivo este que fará a pá apresentar uma torção ao longo do seu comprimento.

Como o BEM considera a força de arrasto sobre a pá nula, é fixado um ângulo de ataque que corresponde à máxima razão $C_{L} / C_{D}$. Como o perfil utilizado no projeto é o NREL $\mathrm{S} 809$, o valor de $\alpha=6,11^{\circ}$ com o correspondente valor $\mathrm{C}_{\mathrm{L}}=$ 0,748. Estes valores são obtidos considerando o valor de $\mathrm{Re}=3 \cdot 10^{5}$ (Butterfield et al., 1992).

Para os ensaios de captura de sinais serão utilizados três conjuntos de pás distintas nos testes, estas diferindo entre si pelo valor da a razão de ponta de pá (tip speed ratio), $\lambda$ : seis, sete e nove.

\subsection{Construção das pás do rotor}

Para construção dos modelos, será utilizada a prototipagem rápida. Esta técnica é definida como um conjunto de processos tecnológicos que permitem a fabricação de modelos físicos tridimensionais a partir de desenhos virtuais projetados no computador. Para fazer tais desenhos são utilizados softwares como CAD, CAE, CAM entre outros. Os desenhos são então transportados do computador para uma máquina responsável pela sua materialização.

A estereolitografia (SLA) é a mais antiga e mais importante técnica de prototipagem rápida, pois supera as demais técnicas de prototipagem rápida pela transparência, maior precisão e melhor acabamento do modelo (MRA, 2013).

Para fabricação das pás do modelo em escala, será utilizada a impressora 3D mostrada na Fig. 3, esta que utiliza da tecnologia SLA. Esta impressora utiliza o método de laminação de folha plástica de PVC (Cloreto de Polivinila). Tal tecnologia consiste em sobrepor lâminas de material plástico, e de forma simultânea corta e cola as mesmas. As dimensões máximas do modelo para impressão são: $160 \mathrm{~mm}, 210 \mathrm{~mm}$ e $135 \mathrm{~mm}$ (X, Y, Z).

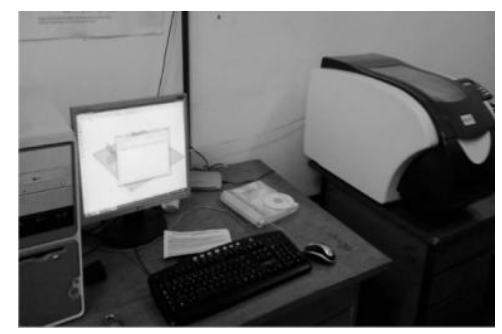

Figura 3. Equipamento de Prototipagem 3D.

\subsection{Montagem do aparato experimental}

Para a obtenção de dados experimentas, deve-se contar com um conjunto de instrumentos de medidas apropriado para obter as principais informações envolvidas no problema estudado: sinal de vibração, rotação, o torque, potência gerada e velocidade dos ventos. Os equipamentos utilizados para conseguir tal objetivo estão apresentados na Fig. 4. 


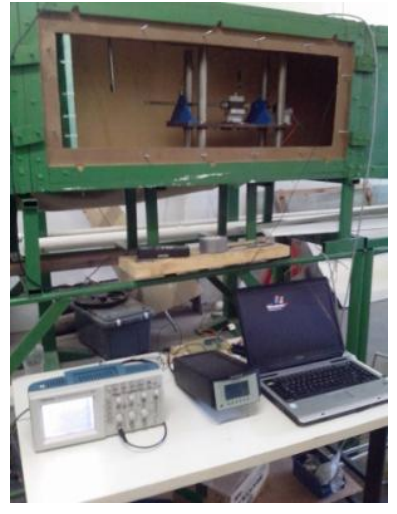

Figura 4. Aparato Experimental

A bancada utilizada foi especialmente construída para testes em escala de turbinas. Trata-se de um dispositivo projetado para operar em túneis de vento dos mais variados tamanhos por possuir ajuste de altura de rotor.

A bancada (conforme pode ser visto na Fig. 5) possui uma placa fixa na parte inferior. Esta é a base do conjunto e nela são fixadas seis colunas com altura máxima de $500 \mathrm{~mm}$. Colunas estas por onde a placa superior móvel correrá podendo ser fixada em diferentes alturas. Na sua placa superior da placa móvel, estão fixados alguns equipamentos: transdutor de torque, motor elétrico, além do eixo da turbina.

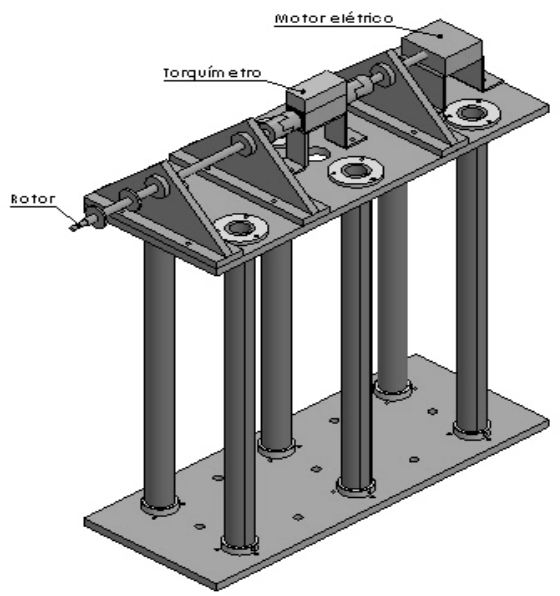

Figura 5. Bancada de testes

Para simular o efeito do ar sobre o rotor eólico construído, foram feitos ensaios em túnel de vento localizado no Laboratório de Aerodinâmica e Mecânica dos Fluidos (LAERO) da Universidade Federal do Ceará.

O túnel de vento é de circuito aberto e possui, na seção de entrada, um bocal com razão de contração de 2:1. Na seção maior de $1,0 \times 1,0 \mathrm{~m}$, e na seção menor $0,50 \times 0,50 \mathrm{~m}$, que faz com que o fluído ao passar por essa região tem um aumento da velocidade devido à diminuição da pressão (Catalano, 1998). Uma representação do túnel de vento é mostrada na Fig.6.

A princípio, seriam realizados testes sem maiores alterações no túnel de vento. Contudo, foram observadas velocidades de corrente livre da ordem de 12 a $16 \mathrm{~m} / \mathrm{s}$. A fim de controlar a velocidade do vento, e minimizar os efeitos de turbulência, foi projetada e construída uma câmara de estabilização com um retificador de fluxo do tipo colmeia. Foram consideradas no dimensionamento da câmara de estabilização as observações de Mehta e Bradshw (1979), segundo as quais o comprimento do favo deve ser de seis a oito vezes o diâmetro médio da célula, e a recomendação de Groff e Alé (2000), que estipula uma faixa para a espessura da parede das células entre 0,5 e 2,0 mm. A colméia foi construída a partir de tubos de PVC.

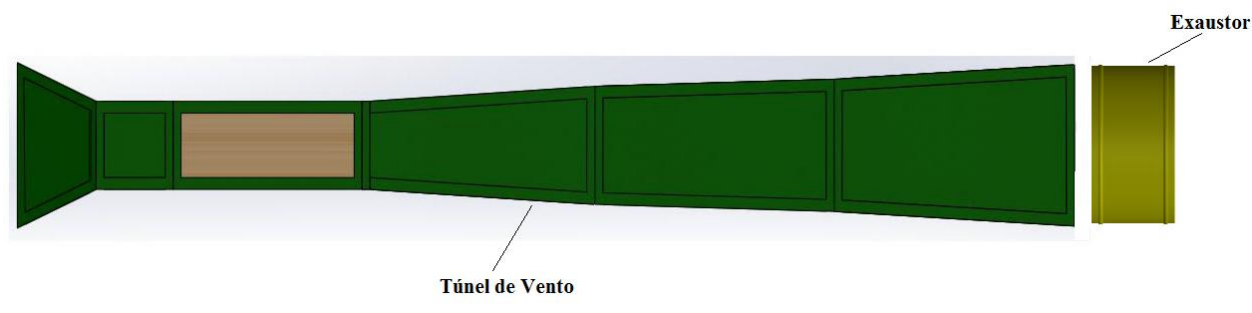

Figura 6. Túnel de Vento 
A seção de saída do túnel é aberta para a atmosfera e é utilizado um exaustor equipamento com um motor de 2HP para movimentar o ar dentro do túnel. Uma representação do exaustor do túnel de vento é mostrada na Fig. 6 . O Exaustor axial trifásico, modelo E100 T8, de dimensões $\phi=100 \mathrm{~cm}$ por $460 \mathrm{~cm}$ de comprimento e vazão de 422 $\mathrm{m}^{3} / \mathrm{min}$.

Para captura de sinal de vibração é necessário um conjunto de equipamentos especial para que a vibração mecânica da turbina eólica, este que é um fenômeno físico real, seja transformando o sinal elétrico proporcional e convertido num formato digital para posterior visualização, armazenamento, processamento e análise. O sistema de medição de vibração utilizado é composto por um acelerômetro, amplificador, osciloscópio e computador. A Fig. 7 esquematiza a montagem do sistema de medição de vibração.

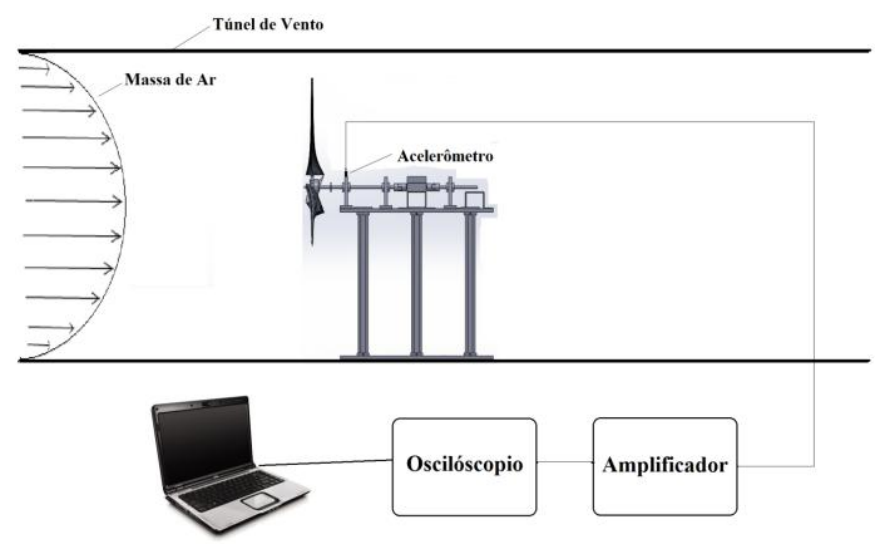

Figura 7. Sistema de captura de sinais.

Para fornecer a ligação direta entre um fenômeno físico e o sistema de aquisição são utilizados sensores ou transdutores. A função destes componentes é converte sinais de grandezas físicas em sinais elétrico (tensões ou correntes) apropriados para os condicionadores de sinais e/ou os equipamentos de aquisição de dados. Especificamente, no presente trabalho foi utilizado um acelerômetro da Brüel\&Kjaer tipo $4384 \mathrm{~V}$.

O sinal capturado pelo acelerômetro necessita de um condicionamento para que o dispositivo de aquisição de dados efetue a medição de forma eficaz e exata. As principais tecnologias de condicionamento de sinal fornecem melhorias distintas tanto no que diz respeito ao desempenho quanto à exatidão de sistemas de aquisição de dados. Para este condicionamento, utilizou-se de um amplificador da Brüel\&Kjaer tipo 2692.

O amplificador tem a função de aumentar o nível de tensão para ampliar à faixa para a qual o dispositivo de aquisição de dados atua, aumentando assim a resolução e sensibilidade da medição. Já os filtros rejeitam ruídos indesejados dentro de uma determinada faixa de frequência.

Uma vez condicionado o sinal vibratório, pode-se fazer a aquisição dos dados. Para isso, conecta-se o condicionador de sinais ao osciloscópio. Este é um instrumento de medição que permite visualizar graficamente sinais elétricos. Na maioria das aplicações, o osciloscópio mostra como é que um sinal elétrico varia no tempo. Os dados mostrados na tela do osciloscópio podem ser ajustados para uma melhor visualização, mas não há modificação dos dados obtidos do condicionador.

Os dados enviados para o osciloscópio podem ser salvos em arquivos de diversos formatos como o TXT, por exemplo. Os dados são enviados para um computador conectado ao osciloscópio.

\section{ANÁLISE ESTATÍSTICA E DE FLUTUAÇÕES}

Uma vez que os dados capturados estiverem disponíveis, dar-se-á inicio à próxima fase do trabalho; análise dos dados. Os primeiro métodos a serem utilizados para caracterizar as falhas serão métodos de análise no domínio do tempo que já são mais tradicionalmente utilizados para análise de vibração.

\subsection{Análise no domínio do tempo}

Os métodos mais simples são a média absoluta $\left(\mathrm{X}_{\mathrm{med}}\right)$, nível global rms $\left(\mathrm{X}_{\mathrm{rms}}\right)$ e o fator de crista $\left(\mathrm{F}_{\mathrm{cr}}\right)$. A média absoluta é dada por (Bezerra, 2004):

$$
X_{\text {med }}=\frac{1}{N} \sum_{i=1}^{N}\left|z_{i}\right|
$$

O nível global rms dá uma estimativa do conteúdo de energia do sinal vibratório, ele é usado para estimar a severidade de vibração proveniente da estrutura de uma máquina ou de fatores externos, sendo mais usado que a média (Mitchell, 1993). O valor do $\mathrm{X}_{\mathrm{rms}}$ é dado por (Bezerra, 2004): 


$$
X_{r m s}=\sqrt{\frac{1}{N} \sum_{i=1}^{N} x_{k}^{2}}
$$
2004):

$\mathrm{O}$ fator de crista é a relação entre o valor de pico $\left(\mathrm{X}_{\text {pico }}\right)$ e o valor rms $\left(\mathrm{X}_{\mathrm{rms}}\right)$, conforme Equação 6.7 (Bezerra,

$$
F_{c r}=\frac{X_{p i c o}}{X_{r m s}}
$$

Os próximos métodos a serem apresentados estão relacionados à densidade de probabilidade; A variância $\left(\sigma^{2}\right)$, assimetria ou skewness $\left(\sigma^{3}\right)$, curtose $\left(\sigma^{4}\right)$ e momento de sexta ordem:

$$
\begin{aligned}
\sigma^{2} & =\frac{\sum_{i=1}^{N}\left(x_{i}-\mu\right)^{2}}{N} \\
\sigma^{3} & =\frac{\sum_{i=1}^{N}\left(x_{i}-\mu\right)^{3}}{N} \\
\sigma^{4} & =\frac{\sum_{i=1}^{N}\left(x_{i}-\mu\right)^{4}}{N} \\
\sigma^{6} & =\frac{\sum_{i=1}^{N}\left(x_{i}-\mu\right)^{6}}{N}
\end{aligned}
$$

\subsection{Análise de flutuações}

Na captura de sinal de vibração utiliza-se de equipamentos especiais e os dados obtidos expressam valores de uma variável (deslocamento, velocidade ou aceleração) em função do tempo. Ao realizar uma série de observações do mesmo mensurando sob as mesmas condições, podemos obter resultados diferentes. Essa variabilidade dos resultados das medições é chamada de flutuação estatística.

A Detrended Fluctuation Analysis (DFA) foi introduzida para estudo de correlações de longo alcance na sequência de DNA (Peng et al., 1994). Seja uma série temporal $\{\mathrm{x}\}$, que possuir valores $x_{i}$, com i variando de 1 a $k$, onde $k$ é o comprimento total da série. Integramos a série utilizando a expressão:

$$
X_{k}=\sum_{i=1}^{k}\left(x_{i}-x_{m}\right)
$$

onde $X_{k}$ é uma nova série obtida pela integração dos valores de $\{\mathrm{x}\}, x_{i}$ é o i-ésimo elemento da série e $x_{m}$ é a média do conjunto de dados:

$$
x_{m}=\frac{1}{k} \sum_{i=1}^{k} x_{i}
$$

O próximo passo é dividir a nova série $X_{k}$ em segmentos não sobrepostos de igual tamanho $\tau$. Os segmentos são chamados de janelas (caixas) representadas por $\mathrm{n}=$ int $(\mathrm{k} / \tau)$. Em cada janela, introduz-se a função de tendência:

$$
Y_{l}=a+b x
$$

onde os coeficientes a e b representam o ajuste linear pelo método dos mínimos quadrados. Por fim, calcula-se a função de flutuação $F(\tau)$ :

$$
F(\tau)=\sqrt{\frac{1}{N_{\tau}} \sum_{i=1}^{N_{\tau}}\left[Y(i)-Y_{\tau}(i)\right]^{2}}
$$

Se for plotado um gráfico $\log \left[\mathrm{F}_{\mathrm{DFA}}\right]$ por $\log (\tau)$, pode-se ajustar uma reta. Esta terá um coeficiente angular $\alpha$ que é o valor do DFA para aquela série. 


$$
F_{D F A}(\tau) \sim \tau^{\alpha}
$$

Se $0<\alpha<0,5$ : a série temporal será antipersistência (autocorrelação negativa). Se há um aumento do passo do tempo de $t_{i}-1$ a $t_{i}$ provavelmente haverá decaimento de $t_{i}$ a $t_{i}+1$. O comportamento parecido se observa para decrescimento de passos. Se > 0,5: corresponde a uma série temporal com comportamento de persistência (autocorrelação positiva). Se há um aumento do passo do tempo de ti-1 a ti provavelmente haverá aumento de $t_{i}$ a $t_{i}+1$. O comportamento parecido se observa para decrescimento de passos. Muitas vezes este tipo de série é chamado de movimento Browniano fracionário $\alpha=0,5$ : corresponde a um movimento Browniano normal e a ausência de efeitos de memória (Batista, 2006).

\section{RESULTADOS E DISCUSSÃO}

A geometria das pás obtida a partir da Eq. (1) à Eq. (5) para os valores de $\lambda$ de projeto $6,7,8$ e 9 são descritas a partir da dependência da corda do perfil e do ângulo de torção como função da dimensão radial ao longo da pá. Os gráficos da Fig. 8 mostram as curvas de afilamento e torção das pás, respectivamente, utilizadas nos testes.

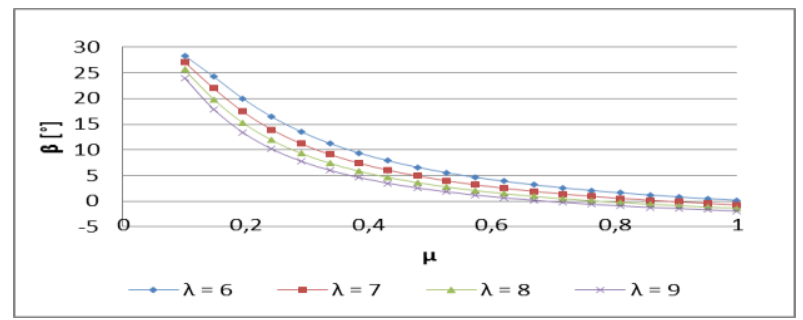

a)

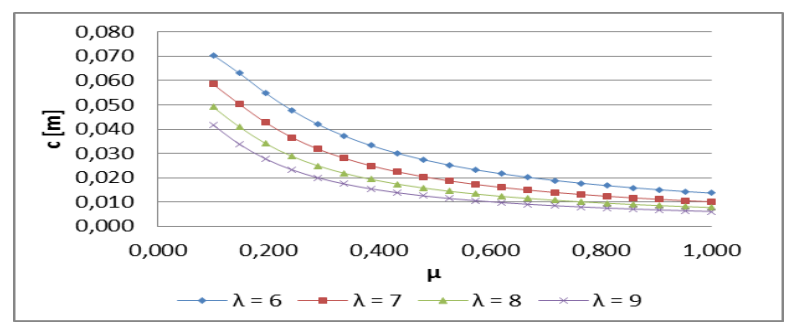

b)

Figura 8. (a) Curvas de torção das pás; (b) curvas de afilamento das pás.

Verifica-se o decréscimo gradual tanto de torção quanto do valor de corda dos perfis utilizados nas pás. Isso garante que em regime de operação variável tenha maior eficiência na extração de energia. Espera-se maior eficiência de cada pá quando esta esteja operando no seu $\lambda$ de projeto, pois o escoamento do ar nos perfis sob tais condições apresenta máxima razão sustentação/arraste.

Até a data de apresentação deste trabalho, só foi possível a construção de três conjuntos de pás: $\lambda_{\mathrm{p}}=6,7 \mathrm{e} 9$. Logo, os testes de desempenho apresentados só contemplam estes três conjuntos. O resultado da prototipagem 3D é mostrado na Fig. 9.

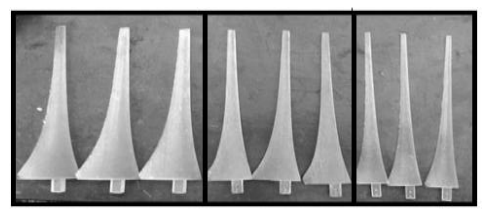

Figura 9. Conjunto de pás Construídas. Da esquerda pra direita $\lambda=6,7 \mathrm{e}=9$

Os sinais foram capturados em testes de captura de sinais com a turbina utilizando um conjunto de pás por vez, variando-se a rotação do equipamento (300 rpm, $600 \mathrm{rpm}, 900 \mathrm{rpm}, 12000 \mathrm{rpm}$ e $1500 \mathrm{rpm}$ ). Os sinais capturados mostraram perfil semelhando aos da Fig. 10.

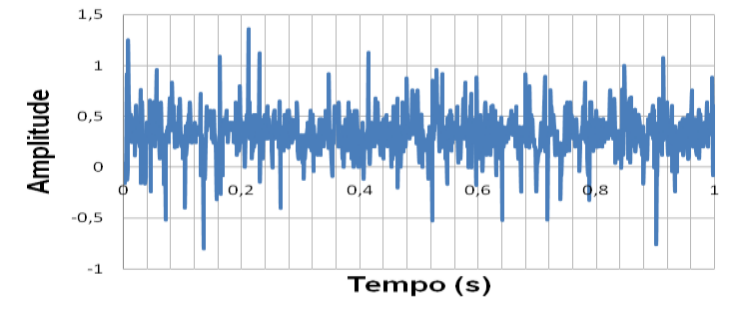

Figura 10. Sinal capturada para turbina eólica de $\lambda_{\mathrm{p}}=9$ à $300 \mathrm{rpm}$

A primeira análise foi aplicar as ferramentas de análise no domínio do tempo nos sinais capturados. A Fig. 11 mostra como os valores dos parâmetros resultantes desta análise variam para cada conjunto de pá para diferentes valores de rotação. 


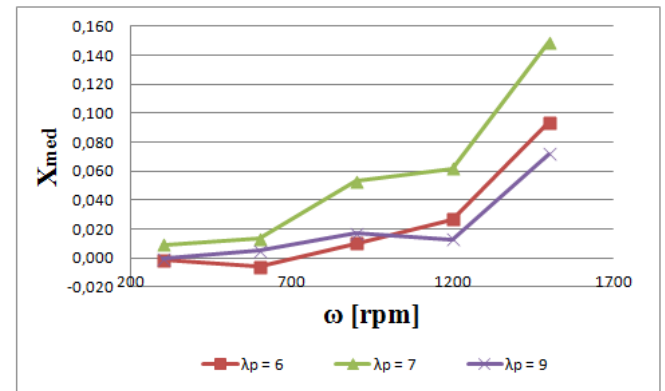

a)

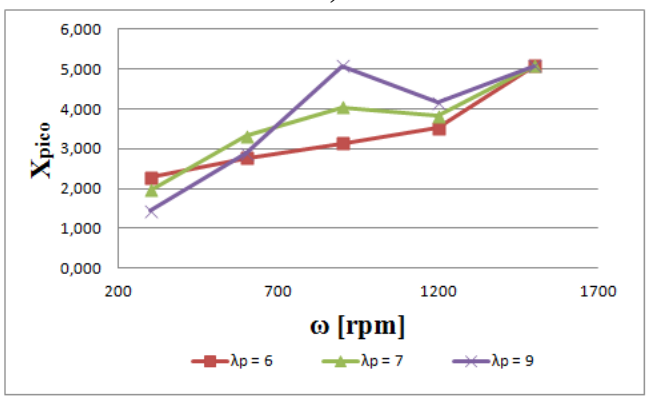

c)

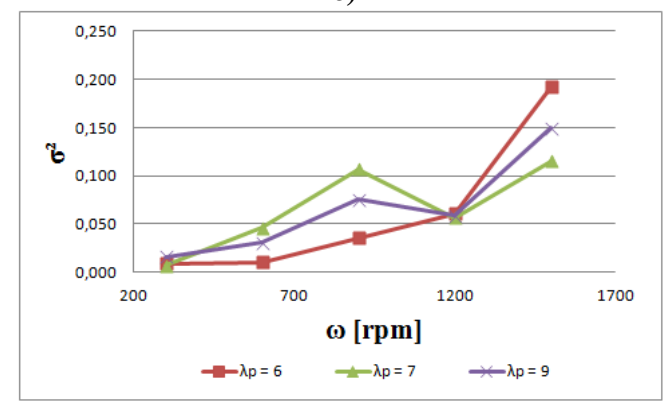

e)

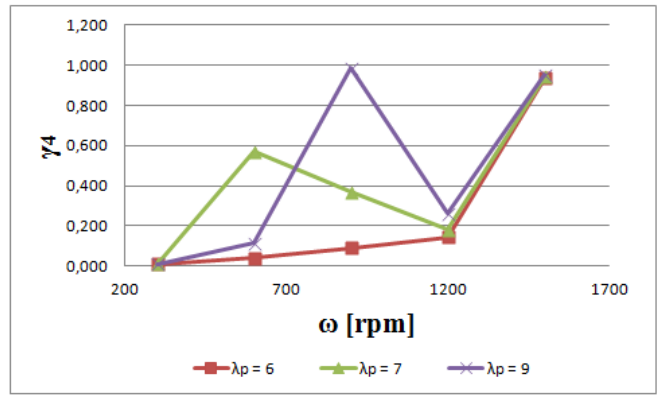

g)

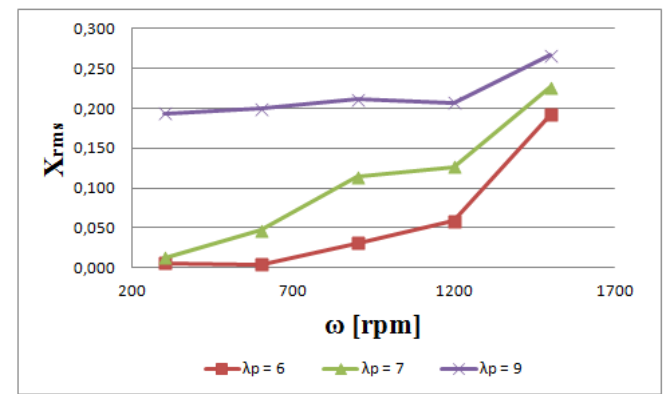

b)

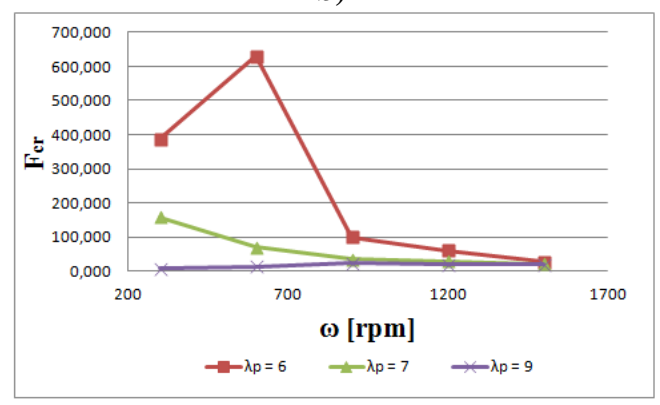

d)

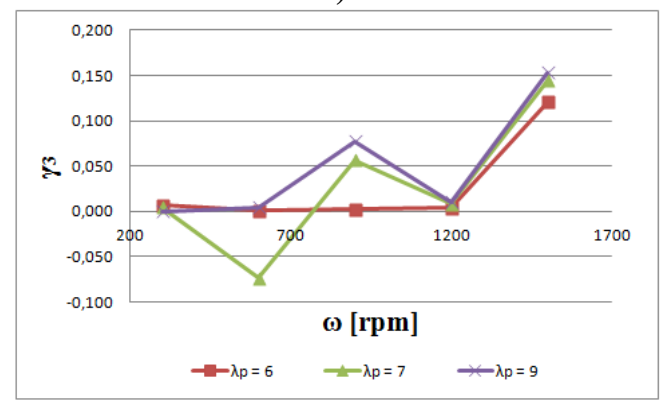

f)

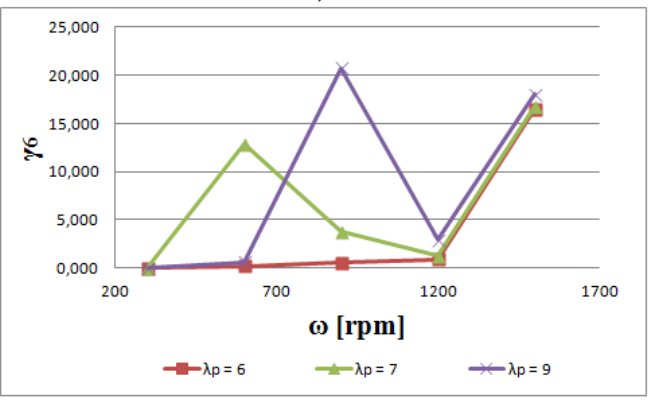

h)

Figura 11. Variação de parâmetros estatísticos em relação à velocidade e $\lambda_{p}$, (a) Média; (b) Índice RMS; (c) Valor de Pico; (d) Fator de Crista; (e) Variância; (f) Assimetria; (g) Curtose; (h) Momento de Sexta Ordem.

Percebe-se que, à medida que a velocidade de rotação aumenta, ou seja, que o tamanho da falha vai progredindo, a média absoluta, o nível RMS, pico máximo crescem. Já o fator de crista decresce. Os parâmetros estatísticos (variância, assimetria, curtose e momento de sexta ordem) crescem à medida que a velocidade de rotação. Observa-se que estes paramentos estatísticos tem uma tendência definida o que caracteriza o sinal de operação de uma turbina eólica sem falhas.

O próximo passo é aplicar o DFA aos sinais capturados. Abaixo é apresentada a Fig. 12 com os sinais capturados e o seu respectivo perfil resultante da análise DFA para o conjunto com $\lambda \mathrm{p}=9$ para diferentes rotações.

Observa-se que, à baixas rotações, os sinais capturados apresentam uma instabilidade correlação entre seus dados apresentando negativa autocorrelação negativa (antipersistência) a $300 \mathrm{rpm}$, passando a autocorrelação positiva (persistência) quando gira a $600 \mathrm{rpm}$. Em seguida, observa-se uma tendência aos valores de $\alpha$ se aproximar de 0,5 com o aumento da rotação (acima de 900 rpm).

Em seguida é apresentada a tab. 1 com os valores de $\alpha$ para os conjuntos de pás utilizados para diferentes valores de rotação e em seguida os sinais capturados. 


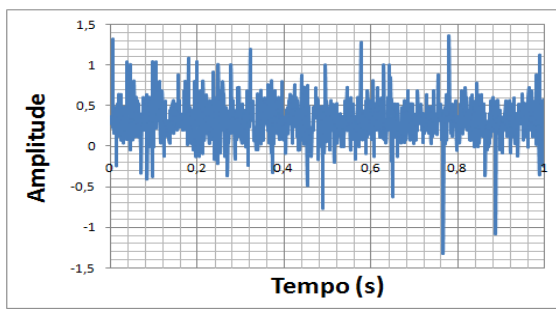

a)

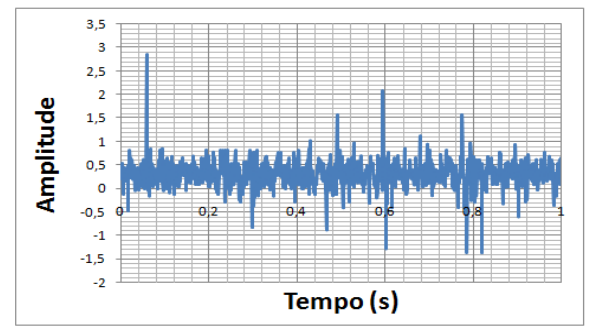

c)

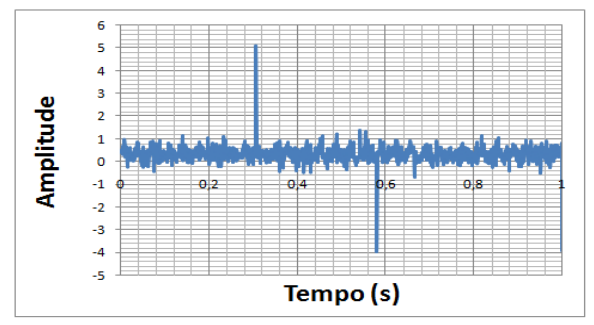

e)

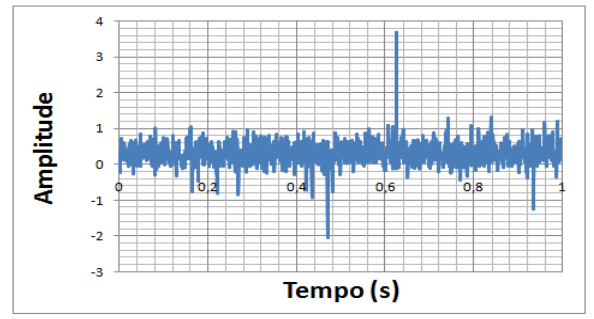

g)

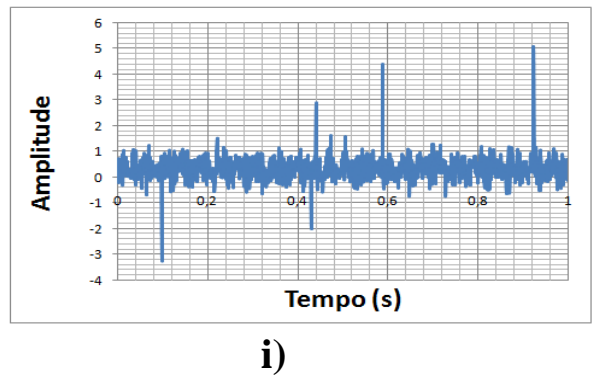

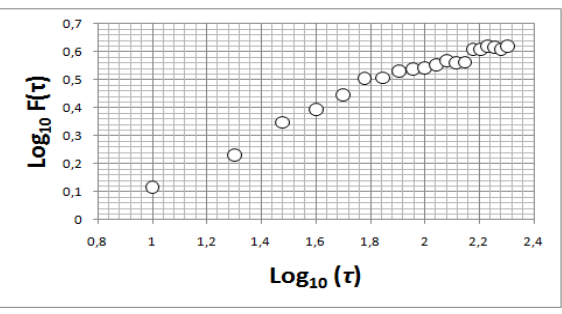

b)

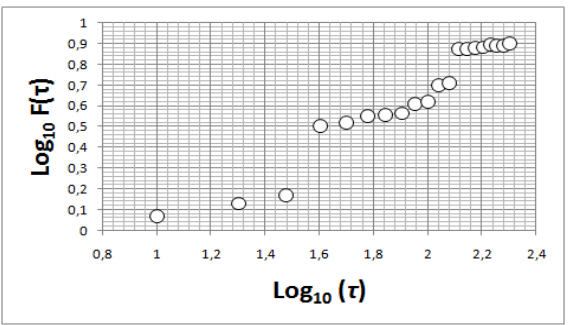

d)

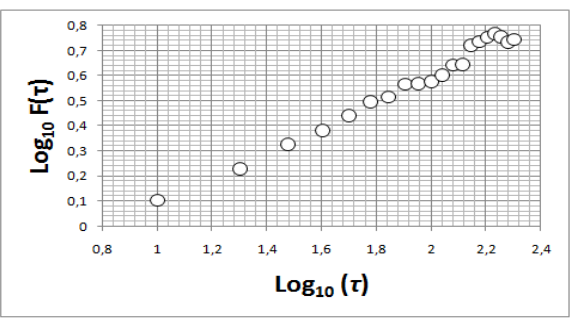

f)

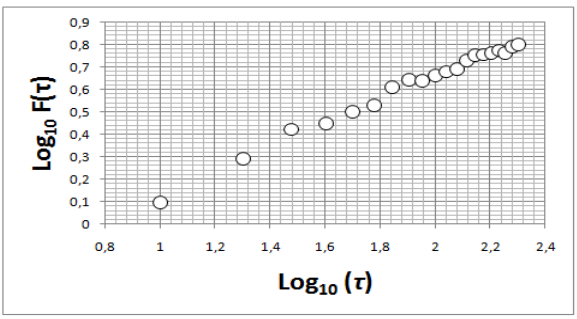

h)

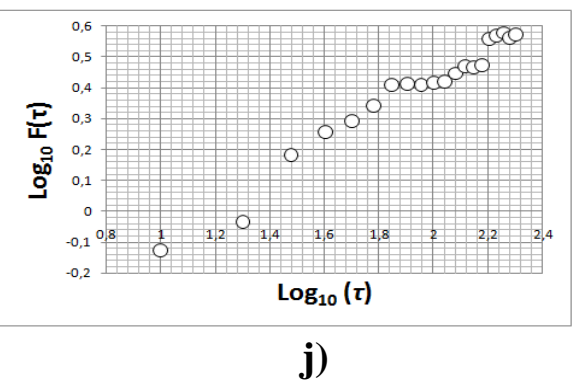

Figura 12. Representação de sinais capturados e curvas DFA para rotor girando com pás de $\lambda p=9$ com diferentes valores de rotação: a) Sinal a $300 \mathrm{rpm}$, b) curva DFA para sinal a $300 \mathrm{rpm}$, c) Sinal a $600 \mathrm{rpm}$, d) curva DFA para sinal a $600 \mathrm{rpm}$, e) Sinal a $900 \mathrm{rpm}$, f) curva DFA para sinal a $900 \mathrm{rp}$, g) Sinal a $1200 \mathrm{rpm}, \mathrm{h}$ ) curva DFA para sinal a $1200 \mathrm{rpm}, \mathrm{g}$ ) Sinal a $1500 \mathrm{rpm}$, h) curva DFA para sinal a $1500 \mathrm{rpm}$.

Tabela 1. Resultados experimentais para $\alpha_{D F A}$ das séries temporais de Torque, potência gerada e rotação.

\begin{tabular}{c|ccccc}
\hline Conjunto de Pás & 300 rpm & $\mathbf{6 0 0} \mathbf{~ r p m}$ & $\mathbf{9 0 0} \mathbf{~ r p m}$ & $\mathbf{1 2 0 0} \mathbf{~ r p m}$ & $\mathbf{1 5 0 0} \mathbf{~ r p m}$ \\
\hline $\boldsymbol{\alpha}_{\mathrm{DFA}}\left(\lambda_{\mathrm{p}}=\mathbf{6}\right)$ & 0,22 & 0,67 & 0,55 & 0,53 & 0,58 \\
$\boldsymbol{\alpha}_{\mathrm{DFA}}\left(\lambda_{\mathrm{p}}=7\right)$ & 0,33 & 0,91 & 0,62 & 0,57 & 0,55 \\
$\boldsymbol{\alpha}_{\mathrm{DFA}}\left(\lambda_{\mathrm{p}}=9\right)$ & 0,38 & 0,73 & 0,53 & 0,52 & 0,53 \\
\hline
\end{tabular}




\title{
5. CONCLUSÃO
}

A utilização de parâmetros estatísticos para análise de vibração foi útil para descrever o perfil de vibração das turbinas eólica para diferentes $\lambda_{\mathrm{p}}$ já que estes apresentaram tendência similar para os três conjuntos de pás utilizadas.

Constatou-se, que a análise de flutuações estatísticas é uma ferramenta que pode ser utilizada para avaliação destas séries temporais, pois seu uso proporcional a descrição da autocorrelação entre os dados capturados, apresentando um perfil de valores de $\alpha$ para os três conjuntos de pás testados.

Conclui-se que a análise de flutuações apresenta rendimento similar à análise no domínio no tempo, pois ambas descreveram o perfil de vibrações para a operação de turbinas eólicas.

\section{AGRADECIMENTOS}

Este trabalho foi financiado pela Fundação Cearense de Apoio ao Desenvolvimento Científico e Tecnológico (FUNCAP) e Coordenação de Aperfeiçoamento de Pessoal de Nível Superior (CAPES).

\section{REFERÊNCIAS}

Anastacio, J. R. B., Sousa, I.D.P., Andrade, C.F., Teixeira, L.P., Albiero, D., Rocha, P.A.C. Aerodynamic Performance Analysis of Small Wind Turbine Blades Using NREL S809 Profile and Various Tip Speed Ratio. In: 22th International Congress of Mechanical Engineering, Ribeirão Preto, SP, 2013.

Burton, T., D. Shape, N. Jenkins, and E. Bossanyi, 2001. Wind Energy handbook. John Wiley \& Sons Ltd.

Butterfield, C. P., Musial, W. P., Scott, G. N., Simms, D.A., 1992. NREL Combined Experimental Final Report - Phase II. National Renewable Energy Laboratory. Colorado, United States.

Catalano, F. M. Projeto, construção e calibração em túnel aerodinâmico de circuito aberto tipo N.P.L. de seção transversal hexagonal. USP: São Carlos, 1998.

Groff, J. E. A.; Alé, J. V. Projeto de túnel de vento subsônico de circuito aberto. Rio Grande do Sul: PUC, 2000.

Leishman, J. G. (2006). Principles of Helicopter Aerodynamics. Cambridge University Press.

Mehta, R. D., Bradshaw, P. Design rules for a small low speed wind tunnels. The Aeronautical Journal of the Royal Aeronautical Society, 1979.

Manwell, J. F., McGowan, J. G, Rogers, A. L., 2006. Wind Energy Explained - Theory, Design and Application. John Wiley \& Sons, Chichester, 2nd edition.

Peng, C. K.; Buldyrev, S.V.; Havlin, S.; Simons, M.; Stanley, H.E; Goldberg, A.L. Mosaic organization of DNA nucleotides. Physical Review E, v. 49, p. 1685-1689, 1994.

Rocha, P. C., Carneiro, F. O., Lopes, A. S., Maia, I. A., \& Modolo, A. B., 2010. Montagem e Testes de uma Bancada para Medição de Desempenho de Aerogeradores em Escala. Revista Brasileira de Ensino de Física, Vol. 32.

\section{RESPONSABILIDADE AUTORAL}

“Os autores são os únicos responsáveis pelo conteúdo deste trabalho”.

\section{COMPARISON BETWEEN STATISTICAL MODELS APPLIED TO VIBRATION ANALYSIS IN SMALL WIND TURBINE}

\author{
José Rafael Bezerra Anastacio, jorafael_bezerra2@hotmail.com ${ }^{1}$ \\ Paulo Alexandre Costa Rocha, paulo.rocha@ufc.br ${ }^{1}$ \\ Francisco Marcilio Vieira Dantas, marciliodantas85@gmail.com ${ }^{1}$ \\ Carla Freitas de Andrade, carla@ufc.br ${ }^{1}$ \\ Roberto de Araújo Bezerra, roberto.bezerra@gmail ${ }^{1}$
}

\begin{abstract}
This paper made a comparative studying of statistical methods applied to the analysis of vibrations in small wind turbines. Two methodologies were addressed: vibration analysis in the time domain and fluctuation analysis. It is used tools such as absolute mean, global average, crest factor, variance, skewness, kurtosis and sixth-order moment. The detrended fluctuation analysis is the tool used for the fluctuation analysis. The vibration signals captured were obtained from tests on small-scale wind turbines that have bladed rotors manufactured with profile NREL S809, $40 \mathrm{~cm}$

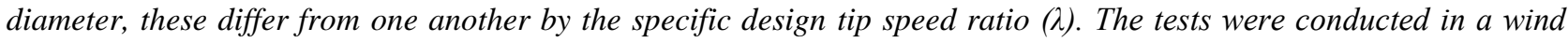
tunnel located at the Laboratory of Aerodynamics and Fluid Mechanics (LAERO), Federal University of Ceará.
\end{abstract}

Keywords: Wind Turbine, Statistics Methods, Fluctuation Analysis, Vibration Control 